\title{
Long-term outcome of catheter ablation and other form of therapy for electrical storm in patients with implantable cardioverter-defibrillators
}

\author{
Stanislaw Morawski ${ }^{1} \cdot$ Patrycja Pruszkowska $^{2} \cdot$ Beata Sredniawa $^{1} \cdot$ \\ Radoslaw Lenarczyk $^{2} \cdot$ Zbigniew Kalarus $^{1}$
}

Received: 12 February 2017 / Accepted: 8 October 2017 / Published online: 24 October 2017

(C) The Author(s) 2017. This article is an open access publication

\begin{abstract}
Purpose Radiofrequency catheter ablation (RFCA) for electrical storm (ES) has become a widely used therapeutic method. Its effectiveness in comparison to other forms of ES treatment is however uncertain.

Methods This single-centre retrospective study investigated the long-term clinical outcome after RFCA for ES and compared long-time effects of ablation to other forms of treatment. The study population consisted of 70 consecutive patients hospitalised between January 2010 and June 2015 due to ES. Patients were recruited for the study if the following criteria were fulfilled: first ES caused by ventricular tachycardia (VT) or ventricular fibrillation (VF), implanted cardioverter defibrillator or cardiac resynchronisation therapy device and left ventricular ejection fraction $<50 \%$. The follow-up data on VT/ES recurrence was obtained from pacemaker/implanted cardioverter defibrillator memory. Data on all-cause mortality was collected during outpatient visits or by telephone contact. Results Of the 70 patients enrolled, $28(40 \%)$ were treated with RFCA (group A) and 42 (60\%) received other forms of treatment for ES (group B). During a mean $( \pm \mathrm{SD}) 864$ (629)
\end{abstract}

Electronic supplementary material The online version of this article (https://doi.org/10.1007/s10840-017-0291-1) contains supplementary material, which is available to authorized users.

Stanislaw Morawski morawski.stan@gmail.com

1 Department of Cardiology, Congenital Heart Diseases and Electrotherapy, School of Medicine with Division of Dentistry in Zabrze, Medical University of Silesia, M.C Sklodowskiej Street 9, 40-055 Zabrze, Poland

2 Silesian Center for Heart Diseases, M.C Sklodowskiej Street 9, 40-055 Zabrze, Poland days of follow-up, death occurred in 4 (14.3\%) patients in the ablation group and in $16(38.1 \%)$ patients treated with other methods $[p=0.03]$. There was no significant between-group difference in VT/VF and ES recurrence. Statistical analysis revealed that the presence of cardiac resynchronisation therapy device during ES, stroke and/or transient ischaemic attack and lower baseline hematocrit level were the multivariate predictors of all-cause mortality.

Conclusions In patients treated with RFCA for ES, all-cause mortality was significantly lower compared to the group treated with other methods.

Keywords Electrical storm - Catheter ablation · Ventricular tachycardia $\cdot$ Ventricular fibrillation $\cdot$ Implantable cardioverter-defibrillator

\section{Introduction}

Heart failure (HF) is a growing medical problem in developed countries, with incidences among adult population reaching up to $2 \%$ [1]. It is well known that HF patients are simultaneously at a significantly higher risk of sudden cardiac death. The recommended and most efficient form of primary and secondary prevention against sudden cardiac death in heart failure patients is the implantation of automatic implantable cardioverter defibrillators (ICD) $[2,3] .3 .5 \%$ of patients with an ICD for primary prevention, and even $10-40 \%$ of those implanted for secondary prevention of SCD, will suffer from the most malignant form of ventricular arrhythmia, known as an electrical storm (ES) $[4,5]$. An electrical storm is defined as the occurrence of three or more distinct episodes of ventricular tachycardia (VT) and/or ventricular fibrillation (VF) within $24 \mathrm{~h}$ [6]. In patients with ICD, its usual presentation involves multiple antitachycardia pacing therapies (ATP) and/or high- 
energy therapies within a short-time period. Management in this challenging group is generally focused on the elimination of potentially reversible causes of electrical instability, haemodynamic stabilisation and (once reversible causes have been excluded or dampened) modification of the arrhythmogenic substrate with catheter ablation of ventricular tachycardia [7-9]. Most of the published data on radiofrequency catheter ablation (RFCA) for ES is based on small series or case reports, mainly describing single treatment method. No large studies have compared RFCA with conservative treatment and their effects on survival. The purpose of this study was to assess the long-term clinical outcomes after RFCA for ES and to compare this method with other forms of treatment for ES.

\section{Methods}

\subsection{Patients and eligibility criteria}

The study population consisted of 70 consecutive patients, who were hospitalised between January 2010 and June 2015 due to the occurrence of an electrical storm. In 68 patients, the arrhythmia responsible for the occurrence of ES was VT; in 2, the ES was due to VF.

Patients were initially recruited for the study if they fulfilled the following criteria: diagnosed with the first electrical storm caused by VT or VF, previous or present implantation of an ICD or cardiac resynchronisation therapy device (CRT-D) during the first ES hospitalisation, and heart failure with left ventricular ejection fraction (LVEF) $<50 \%$ (assessed by transthoracic echocardiography). Patients were excluded if they were suffering from inherited arrhythmogenic diseases (long QT syndrome, Brugada syndrome, catecholaminergic polymorphic ventricular tachycardia, short QT syndrome arrhythmogenic right ventricular cardiomyopathy), had suffered from a previous electrical storm, had been previously treated with VT ablation or were aged $<18$ years old. The clinical characteristics of the total study population of patients with a particular emphasis on age, sex, comorbidity, basic electrocardiographic, echocardiographic parameters, the type of cardiomyopathy and the type of prevention causing ICD/CRT-D implantation are shown in Table 1 . To evaluate the effect of catheter ablation for the long-term prognosis of patients treated because of an electrical storm, the research group was divided depending on ablation procedure (group A) or no ablation procedure (group B) during primary hospitalisation. The clinical characteristics of group A (28 patients) were compared with those of the remaining study population (42 patients; group B) as show in Table 1.

During hospitalisation, if presenting VT episodes were recorded based on 12-lead ECG and/or 12-lead Holter ECG, the corresponding ECG morphologies were defined as clinical
VT. Coronary angiography was performed in 44 patients in whom myocardial ischaemia was suspected. In the remaining population, coronary arteriography was performed in referral hospitals (5 patients) and in the previous 12 months ( 9 patients).

In 7 patients, this was not conducted because of no clinical suspicion of myocardial ischaemia or because of nonischaemic cardiomyopathy (5 patients). Percutaneous coronary intervention was performed in 18 patients.

No additional non-pharmacological beta blockade procedures were performed (such as left stellate ganglion blockade). No overdrive atrial pacing was used to tide over the storm in any of the patients. Patients underwent standard treatment in terms for reversible reasons of ES. In retrospective analysis during primary hospitalisation, 28 patients were treated using the catheter ablation. A RFCA was performed in all patients who underwent the invasive electrophysiological study.

\subsection{Electrophysiological study and ablation strategy}

Written informed consent was obtained from all patients before RFCA. All procedures were performed under local anaesthesia. In all cases, retrograde femoral access was used. Mapping was performed using the three-dimensional electro-anatomical mapping system Carto 3 (Biosense Webster, Diamond Bar, CA, USA) and a saline irrigated tip catheter NaviStar ThermoCool (Biosense Webster). In all cases, endocardial mapping was routinely undertaken. An initial bolus of heparin, in dose $80 \mathrm{IU} / \mathrm{kg}$ of patient body weight, was administered after the electrode was positioned in the left ventricle. Every $15 \mathrm{~min}$, the activating clothing time (ACT) was measured and an additional heparin bolus was administered to maintain ACT above $250 \mathrm{~s}$. In all patients in sinus rhythm (SR), three-dimensional left ventricular bipolar voltage maps were constructed. A dense scar was defined as an area of local bipolar peak-to-peak voltage of $<0.5 \mathrm{mV}$ and a border zone $<1.5 \mathrm{mV}$. In patients with haemodynamically stable VT, arrhythmia was induced by programmed stimulation and a three-dimensional activating map was obtained as in patients with an incessant VT or if arrhythmia occurred spontaneously.

Targets for ablation were determined as mid-diastolic potentials, areas of slow conductions, or identifying isthmus and exit zones with prolonging time stimulus to QRS in sinus rhythm (SR) patients using pacemapping. During VT, the target for application was determined in areas central to the exit zone and identified by entrainment. Radiofrequency current was delivered with an open irrigated tip catheter with a $30 \mathrm{ml} /$ min saline flow at a power setting of 30 up to $50 \mathrm{~W}$ and a temperature limit of $45^{\circ} \mathrm{C}$. After ablation, programmed stimulation with up to three extra stimuli was performed. The success was defined as non-inducibility of clinical VT. In cases of inducibility, another form of sustained VT, additional 
Table 1 Baseline clinical and demographic characteristics of the study population, ablated (group A) and no ablated (group B) group

\begin{tabular}{|c|c|c|c|c|}
\hline Group characteristics & $N=70$ & Group A $(n=28)$ & Group B $(n=42)$ & $p$ \\
\hline Gender (M/F) & $64 / 6$ & $26 / 2$ & $38 / 4$ & 0.73 \\
\hline Age & $64.71 \pm 10.71$ & $65.75 \pm 8.93$ & $64.02 \pm 11.8$ & 0.51 \\
\hline BMI $\left(\mathrm{kg} / \mathrm{m}^{2}\right)$ & $27.59 \pm 3.72$ & $28.66 \pm 3.65$ & $26.76 \pm 3.61$ & 0.04 \\
\hline NYHA class & $2.16 \pm 0.69$ & $2.16 \pm 0.62$ & $2.17 \pm 0.75$ & 0.97 \\
\hline NYHA $\geq 2$ & $57(81.4 \%)$ & $24(85.7 \%)$ & $33(78.6 \%)$ & 0.46 \\
\hline QRS duration (ms) & $154 \pm 39$ & $159.71 \pm 40.86$ & $150.23 \pm 38.15$ & 0.33 \\
\hline Native QRS duration $(\mathrm{ms})^{\mathrm{a}}$ & $130.59 \pm 28.33$ & $140.61 \pm 30.42$ & $122.0 \pm 23.88$ & 0.04 \\
\hline Paced QRS duration $(\mathrm{ms})^{\mathrm{b}}$ & $183.52 \pm 30.21$ & $194.1 \pm 34.9$ & $178.48 \pm 27.17$ & 0.18 \\
\hline Heart rate/min & $68.14 \pm 11.44$ & $66.96 \pm 10.79$ & $69.93 \pm 11.92$ & 0.49 \\
\hline Ventricular stimulation & $31(44.3 \%)$ & $10(35.7 \%)$ & $21(50 \%)$ & 0.24 \\
\hline $\operatorname{LVEF}(\%)$ & $26.2 \pm 7.31$ & $27.11 \pm 6.27$ & $25.59 \pm 7.95$ & 0.40 \\
\hline LVEDD (mm) & $67.91 \pm 8.33$ & $67.73 \pm 8.15$ & $68.03 \pm 8.55$ & 0.89 \\
\hline $\operatorname{LVESD}(\mathrm{mm})$ & $55.78 \pm 11.23$ & $53.75 \pm 10.86$ & $57.2 \pm 11.42$ & 0.25 \\
\hline $\mathrm{EDV}(\mathrm{ml})$ & $237.96 \pm 78.26$ & $233.75 \pm 80.99$ & $241.19 \pm 77.53$ & 0.75 \\
\hline $\mathrm{ESV}(\mathrm{ml})$ & $175.63 \pm 74.51$ & $164.93 \pm 69.65$ & $182.76 \pm 78.44$ & 0.49 \\
\hline MVR-severe & $2(2.9 \%)$ & $0(0 \%)$ & $2(4.8 \%)$ & 0.24 \\
\hline $\mathrm{LA}(\mathrm{mm})$ & $45.25 \pm 6.82$ & $46.52 \pm 6.94$ & $44.45 \pm 6.72$ & 0.26 \\
\hline Implantation of ICD/CRTD in secondary prevention & $42(60 \%)$ & $19(67.9 \%)$ & $23(54.8 \%)$ & 0.28 \\
\hline Secondary prevention due to VF/SCA & $19(27.1 \%)$ & $9(32.1 \%)$ & $10(23.8 \%)$ & 0.45 \\
\hline During the storm, the presence of an implanted CRT-D & $24(34.3 \%)$ & $8(28.6 \%)$ & $16(38.1 \%)$ & 0.42 \\
\hline During the storm, the presence of an implanted ICD & $41(58.6 \%)$ & $18(63.3 \%)$ & $23(54.8 \%)$ & 0.44 \\
\hline Ischemic cardiomyopathy & $57(81.4 \%)$ & $25(89.3 \%)$ & $32(76.2 \%)$ & 0.17 \\
\hline MI & $54(77.1 \%)$ & $23(82.1 \%)$ & $31(73.8 \%)$ & 0.42 \\
\hline Previous myocardial infarction treated conservatively & $39(55.7 \%)$ & $20(71.4 \%)$ & $19(45.2 \%)$ & 0.03 \\
\hline Number of MI & $1.23 \pm 1.04$ & $1.18 \pm 0.86$ & $1.26 \pm 1.14$ & 0.74 \\
\hline Angioplasty other than MI & $33(47.1 \%)$ & $14(50 \%)$ & $19(45.2 \%)$ & 0.70 \\
\hline Coronary artery bypass grafting & $13(18.6 \%)$ & $8(28.6 \%)$ & $5(11.9 \%)$ & 0.08 \\
\hline Complete revascularisation after angiography & $43(61.4 \%)$ & $19(67.9 \%)$ & $24(57.1 \%)$ & 0.36 \\
\hline Arterial hypertension & $44(62.9 \%)$ & $16(57.1 \%)$ & $28(66.7 \%)$ & 0.42 \\
\hline Diabetes type 2 & $25(35.7 \%)$ & $12(42.9 \%)$ & $13(31 \%)$ & 0.32 \\
\hline Chronic kidney disease & $26(37.1 \%)$ & $8(28.6 \%)$ & $18(42.9 \%)$ & 0.23 \\
\hline Stroke/transient ischaemic attack & $12(17.1 \%)$ & $4(14.3 \%)$ & $8(19 \%)$ & 0.61 \\
\hline Chronic obstructive pulmonary disease & $7(10 \%)$ & $2(7.1 \%)$ & $5(11.9 \%)$ & 0.52 \\
\hline Atrial fibrillation & $31(44.3 \%)$ & $13(46.4 \%)$ & $18(42.9 \%)$ & 0.77 \\
\hline Hyperthyroidism & $8(11.4 \%)$ & $4(14.3 \%)$ & $4(9.5 \%)$ & 0.55 \\
\hline Amiodarone & $32(45.7 \%)$ & $10(35.7 \%)$ & $22(52.4 \%)$ & 0.18 \\
\hline Angiotensin-converting-enzyme inhibitors & $60(85.7 \%)$ & $24(85.7 \%)$ & $36(85.7 \%)$ & 1.0 \\
\hline Beta-blockers & $70(100 \%)$ & $28(100 \%)$ & $42(100 \%)$ & 1.0 \\
\hline Class I antiarrhythmic agents & $3(4.3 \%)$ & $2(7.1 \%)$ & $1(2.4 \%)$ & 0.34 \\
\hline Mineralocorticoid receptor antagonist & $60(85.7 \%)$ & $25(89.3 \%)$ & $35(83.3 \%)$ & 0.49 \\
\hline Loop diuretics & $52(74.3 \%)$ & $21(75 \%)$ & $31(73.8 \%)$ & 0.91 \\
\hline Thiazides & $3(4.3 \%)$ & $1(3.6 \%)$ & $2(4.8 \%)$ & 0.81 \\
\hline Digitalis & $8(11.4 \%)$ & $3(10.7 \%)$ & $5(11.9 \%)$ & 0.88 \\
\hline Lipid-lowering statin drugs & $62(88.6 \%)$ & $26(92.9 \%)$ & $36(85.7 \%)$ & 0.36 \\
\hline Insulin & $7(10 \%)$ & $4(14.3 \%)$ & $3(7.1 \%)$ & 0.34 \\
\hline Oral antidiabetic drugs & $13(18.6 \%)$ & $7(25 \%)$ & $6(14.3 \%)$ & 0.27 \\
\hline Sedatives & 0 & & & \\
\hline Anaesthetics & 0 & & & \\
\hline
\end{tabular}

$B M I$ body mass index, $C R T-D$ cardiac resynchronization therapy defibrillator, $E D V$ end-diastolic volume, $E S V$ end-systolic volume, $I C D$ implantable cardioverter-defibrillator, $L A$ left atrium, $L V E D D$ left ventricular end-diastolic diameter, $L V E F$ left ventricular ejection fraction, $L V E S D$ left ventricular end-systolic diameter, $M I$ myocardial infarction, MVR mitral valve regurgitation, NYHA class New York Heart Association Class, SCA sudden cardiac arrest, $V F$ ventricular fibrillation

${ }^{a}$ Native QRS duration — duration of QRS complex in patients without ventricular stimulation

${ }^{\mathrm{b}}$ Paced QRS duration — duration of QRS complex in patients with ventricular stimulation

mapping and ablation was performed according to the protocol described above. Apart from intra-procedural anticoagulation with unfractionated heparin, in every patient, a standard prophylactic dose of low-molecular-weight heparin was administered in the early post-procedural period. Further anticoagulation was carried out with the use of vitamin $\mathrm{K}$ antagonists, which were instituted on the first post-operative day, and continued for 1 month, with the target INR between 2 and 3. This therapy was subsequently withdrawn, if long-term anticoagulation was not required due to other reasons. 


\subsection{Follow-up}

The majority of the patients $(65 ; 92.9 \%)$ already had implanted ICD $(41 ; 58.6 \%)$ or CRTD $(24 ; 34.3 \%)$ before primary admission. In the rest, ICD $(5 ; 7.1 \%) / C R D T(0 ; 0 \%)$ devices were implanted before the discharge.

The follow-up date for VT/ES recurrence/mortality (average 864 days; mean 706 days, SD 629) was obtained in our pacemaker/ICD outpatients' clinic through interrogation of the devices, remote monitoring (RM), subsequent hospitalisations and telephone contact.

\subsection{Statistical analysis}

Continuous variables are described as mean \pm standard deviation (SD), or median and range for skewed distributions. Categorical variables are described as numbers and percentages. Between-group comparisons were performed with Student's $t$ test or the $\chi^{2}$ test as appropriate. Univariate and multivariate analyses were performed using a Cox proportional hazards model, with hazard ratio (HR) and $95 \%$ confidence intervals $(\mathrm{CI})$ reported. Two multivariate Cox regression models were constructed to predict long-term mortality. The first tested parameters which had significance in the univariate model $<0.05$ (model 1 ), and the second included variables with $p<0.1$ (model 2). Some variables (e.g. creatinine level) were not included into multivariate models due to redundancy with other parameters already included (chronic kidney disease in this case).

Long-term success (i.e. time to VT recurrence, time to ES recurrence and time to death) was also examined in a survival analysis framework. Time-to-event survival curves were graphed for different patient subgroups using the KaplanMeier estimation technique. Differences between strata were assessed by a log-rank test.

The study was carried out in accordance with the Declaration of Helsinki and International Committee on Harmonization guideline for good clinical practice.

\section{Results}

Seventy consecutive patients ( $91.4 \%$ male, age $[64.71 \pm 10.71]$ years; LVEF $26.2 \% \pm 7.31 \%$ ), the majority with ischaemic cardiomyopathy $(81.43 \%)$ and ES, constituted our study population. As described in the "Methods" section, patients were divided into two groups according to execution of catheter ablation during the primary episode of ES. Among the groups, no significant difference in baseline clinical and electrocardiography characteristics were observed except BMI (group A: $28.66 \pm 3.65$ vs. group B: $26.76 \pm 3.61$; $p=0.04)$, myocardial infarction treated non-invasively (20 [71.4\%] vs. 19 [45.2\%]; $p=0.03$ ) and duration of native QRS respectively (group A: $140.61 \pm 30.42 \mathrm{~ms}$ vs. group B: $122.0 \pm 23.88 \mathrm{~ms} ; p=0.04$ ) (Table 1 ).

In group A, the majority of cases showed no clear cause of ES during primary hospitalisation ( $n=24$ [85.71\%]), in contrast to group $\mathrm{B}$, where nearly half of the patients had clear causes of ES ( $n=22$ [53.4\%]). In group B, RFCA was abandoned in 20 patients during hospitalisation despite undetected and potentially reversible cause of electrical instability, due to the disappearance of arrhythmia as a result of the intensification of antiarrhythmic therapy (increased doses of $\beta$-blockers (20 patients) and to the inclusion of treatment with antiarrhythmic class III drugs (14 patients; 13 with amiodarone, 1 with sotalol) (Table 2). After the first ES, 28 patients underwent a catheter ablation procedure. Nine $(32.1 \%)$ patients from group A and 13 (31\%) from B group underwent subsequent ablation during the follow-up because of VT and ES recurrence $(p=0.92)$. Importantly, no statistical differences were observed in terms of VT (group A: 16 [57.1\%]; group B: 31 [73.8\%]; $p=0.15$ ) and ES (group A: 9 [32.1\%]; group B: 19 [45.2\%]; $p=0.28$ ) recurrence in both groups. However, there was significantly lower all-cause mortality in group A (4 [14.3\%] vs. 16 [38.1\%]; $p=0.03$ ) (Table 3). Significant differences in survival between ablated and nonablated groups are shown in Fig. 1. At the end of the ablation procedures, 23 patients underwent programmed electrical
Table 2 Primary episode of ES - causes

\begin{tabular}{llll}
\hline & $\begin{array}{l}\text { Group A } \\
(n=28)\end{array}$ & $\begin{array}{l}\text { Group B } \\
(n=42)\end{array}$ & $p$ \\
\hline No potentially reversible cause & $24(85.7 \%)$ & $20(47.6 \%)$ & $<0.01$ \\
Acute coronary syndrome & $1(3.6 \%)$ & $5(11.9 \%)$ & 0.23 \\
Significant stenosis of the coronary artery requiring & $4(14.3 \%)$ & $16(38.1 \%)$ & 0.03 \\
$\quad$ intervention & $0(0 \%)$ & $3(7.1 \%)$ & 0.15 \\
Infective endocarditis & $0(0 \%)$ & $2(4.8 \%)$ & 0.25 \\
Dyselectrolytemia & $0(0 \%)$ & $1(2.4 \%)$ & 0.42 \\
Discontinuation of medication & $0(0 \%)$ & $1(2.4 \%)$ & 0.42 \\
Exacerbation of heart failure & $0(0 \%)$ & $1(2.4 \%)$ & 0.42 \\
Decompensated hyperthyroidism & $1(3.6 \%)$ & $7(16.7 \%)$ & 0.09 \\
More than 1 cause & & & \\
\hline
\end{tabular}


Table 3 Long-term treatment effect of ES in study population, ablated (group A) and no ablated (group B) group

\begin{tabular}{|c|c|c|c|c|}
\hline & $N=70$ & Group A $(n=28)$ & Group B $(n=42)$ & $p$ \\
\hline $\begin{array}{l}\text { Ventricular tachycardia/ventricular } \\
\text { fibrillation recurrence }\end{array}$ & $47(67.1 \%)$ & $16(57.1 \%)$ & $31(73.8 \%)$ & 0.15 \\
\hline Recurrence of an electrical storm & $28(40 \%)$ & $9(32.1 \%)$ & $19(45.2 \%)$ & 0.28 \\
\hline Number of subsequent electrical storms & $0.8 \pm 1.48$ & $0.43 \pm 0.69$ & $1.05 \pm 1.79$ & 0.09 \\
\hline In-hospital death & $4(5.7 \%)$ & $1(3.6 \%)$ & $3(7.1 \%)$ & 0.54 \\
\hline All-cause death & $20(28.6 \%)$ & $4(14.3 \%)$ & $16(38.1 \%)$ & 0.03 \\
\hline Organ heart transplantation & $1(1.4 \%)$ & $1(3.6 \%)$ & $0(0 \%)$ & 0.22 \\
\hline $\begin{array}{l}\text { Composite of all-cause death, electrical } \\
\text { storm recurrence and ventricular } \\
\text { tachycardia/ventricular fibrillation recurrence }\end{array}$ & $57(81.4 \%)$ & $21(75 \%)$ & $36(85.7 \%)$ & 0.27 \\
\hline
\end{tabular}

stimulation. In 2 of those patients, non-clinical VTs were inducible. There was no inducible clinical VT at the end of the procedure. Five patients (17.9\%) were not tested due to haemodynamic instability after CA.

Analysis of the early post-operative period ( $<30$ days) revealed a non-significantly $(p=0.16)$ higher rate of VA recurrence rates in the group which underwent RFCA (7 [25\%]) vs. the conservative group (5[12\%]), respectively. However, subanalysis of the late ( $>30$ days postoperatively) period only, a significantly $(p=0.02)$ lower rate of recurrences was found in the ablated group $(46.4 \%)$ vs. in the non-ablated group (75\%; 2 patients from the non-ablated group died during the blanking period, and consequently, they were not included in the analysis).

Variables evaluated in the univariate analysis were as follows: age, gender, BMI, NYHA class, LVEF, severe MVR, implantation of ICD/CRTD in secondary prevention of SCA, presence of an implanted CRT-D (during the storm), ischemic cardiomyopathy, previous myocardial infarction treated conservatively, complete revascularisation after angiography, diabetes type 2, chronic kidney disease, stroke and/or transient ischaemic attack, chronic obstructive pulmonary disease, atrial fibrillation, haematocrit level, C-reactive protein level, creatinine level, GFR, NT-pro-BNP level, potentially reversible causes of ES, primary RFCA procedure, VT recurrence and ES recurrence.

The univariate analysis revealed that severe mitral valve regurgitation (MVR), presence of CRT-D during ES, diabetes type 2 , stroke and/or a transient ischaemic attack, a lower baseline haematocrit level, a higher NT-pro-BNP level, a higher C-reactive protein (CRP) level and infective endocarditis as a potentially reversible cause of ES were significant prognostic predictors for all-cause mortality. Further details are provided in Table S1 in the Supplementary Appendix.

The multivariate Cox regression model, incorporating parameters which had significance in the univariate model $<0.05$ as covariates (model 1), demonstrated that both the presence of CRT-D during ES and stroke and/or a transient ischaemic attack were independently associated with the increased risk of total mortality (HR 7.99, 95\%CI 2.35-27.16; $p=0.001$; and HR 4.78, 95\%CI 1.43-15.93; $p=0.01$, respectively). In the second model (including all variables with $p<0.1$ ), the presence of CRT-D during ES (HR 6.83, 95\%CI $1.93-24.24 ; p=0.003$ ), stroke and/or a transient
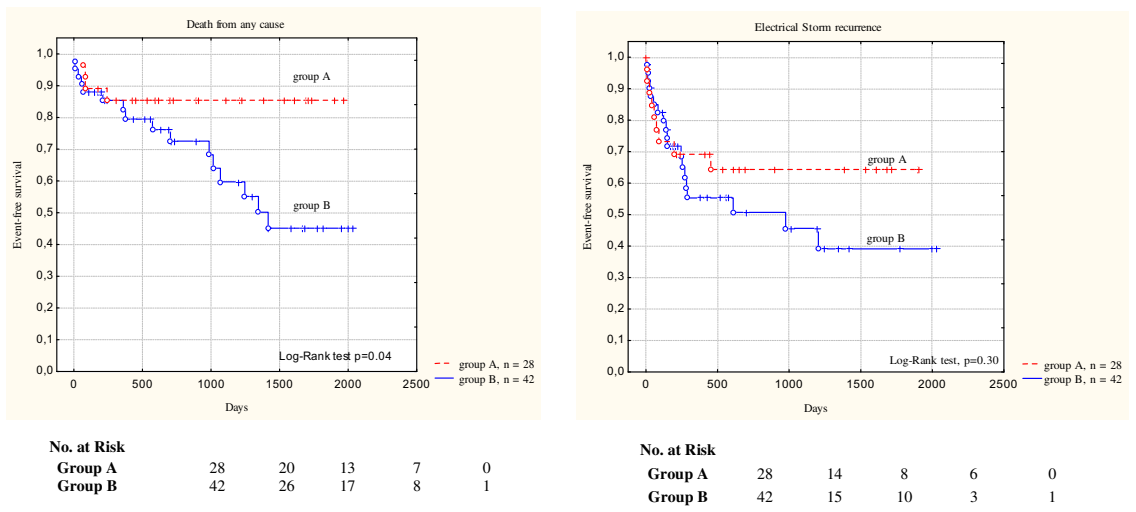

Fig. 1 Kaplan-Meier event-free survival estimates in the patient population during follow-up (group A vs. group B). The Kaplan-Meier curve shows a difference between group A (RFCA treatment in primary hospitalisation) and group B (no-RFCA in primary hospitalisation) in

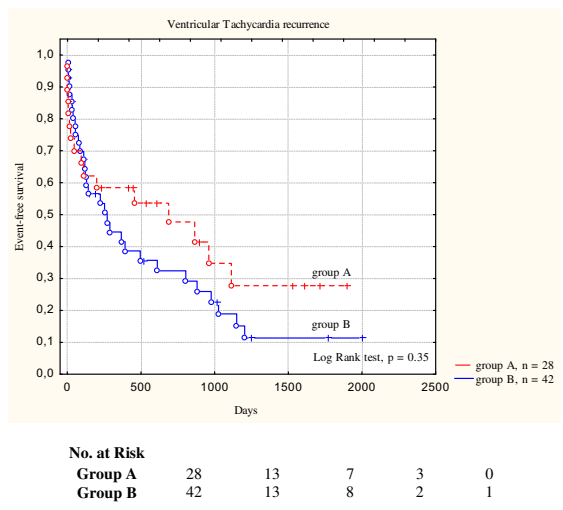

terms of total mortality, electrical storm recurrence and ventricular tachycardia recurrence at $864 \pm 629$ days of follow-up. A statistically significant difference in terms of total mortality is observed (log-rank test, $p=0.04$ ) 
ischaemic attack (HR 9.86, 95\%CI 1.71-56.65; $p=0.01)$ and a lower baseline haematocrit level (HR 0.84, 95\%CI 0.72 $0.99 ; p=0.04$ ) were all independently associated with the higher risk of all-cause mortality. A thorough analysis of these multivariates analyses (both models 1 and 2) is also provided in Table S1 in the Supplementary Appendix.

\section{Discussion}

Recurrent ventricular arrhythmias, which as they are the cause of electrical storms, are the most common problem among ICD/CRT-D recipients. In our study, catheter ablation in such patients was more effective than any other form of therapy in reducing death at any time. Despite the interdisciplinary approach to the treatment, long-term mortality remains high. In our study, more than a quarter of patients died during the course of the study, with the majority of deaths taking place during a few months after an episode of electrical storm. This is concordant with several studies, which have shown that ES occurrence in patients with ICD/CRT-D is an independent risk factor for death, with an increase in risk of at least 2-3-fold in long-term follow-up. The risk is highest in the first 3 months post an electrical storm episode [5, 10-14]. For many years, consensus statements and guidelines have recommended the use of catheter ablation (CA) when antiarrhythmic therapy does not prevent recurrences of ventricular arrhythmias; however, these recommendations have been largely based on expert opinion and non-randomised case series [15-17]. These recommendations were in force up until the publication of the 2015 ESC guidelines for the management of patients with ventricular arrhythmias and the prevention of sudden cardiac death where urgent catheter ablation is recommended (classification of recommendation II B) in patients with scar-related heart disease with incessant VT or electrical storm [18]. However, these guidelines were mainly created based on the Carbucicchio et al. study, where catheter ablation for the management of ES was evaluated in detail [9]. The authors included 95 patients with structural heart disease and electrical storm refractory to antiarrhythmic drug therapy. At a median followup of 22 months, ES recurred in $8 \%$ and cardiac mortality was significantly higher in patients in whom at least one clinical VT could not be abolished, when compared with patients after successful CA defined as non-inducible VT in programmed electrical stimulation. Similar findings were reported by Nayyar et al. [14] in their meta-analysis of 39 publications including a total number of 471 patients with structural and non-structural heart disease, who underwent invasive ablation management of ventricular arrhythmia (VA) storm with total follow-up $61 \pm 37$ weeks. The odds of death were found to be four times higher after a failed procedure, compared to those with a successful procedure (95\%CI 2.04-8.01; $p<0.001$ ). The value of information from our study is that it focuses on patients with structural heart disease and with a first episode of ES, excluding those who had already undergone previous RFCA. Our trial provides evidence that catheter ablation is the most effective first-line life-saving therapy for ES and should not be used only as a bailout therapy. Interestingly, no significant benefit of RFCA with respect to composite of all-cause death, electrical storm recurrence and ventricular tachycardia/ventricular fibrillation recurrence was observed in the group treated with RFCA, compared with those in the remaining population. We hypothesise that the observed reduction of all-cause mortality in the RFCA group (despite lack of statistical significance in recurrence rates) was due to trigger and substrate modification caused by ablation. This modification in turn might have led to different arrhythmias (with more benign characteristics: slower and with lower burden). We can speculate that tissue oedema, which is created during ablation, could render a new reentrant circuit in the surrounding myocardium responsible for creation of new non-clinical VT (e.g. with lower cycle and burden). Indeed, our study has shown a high rate of early VA recurrence after RFCA procedure only within the 30 days after procedure compared with the conservative group ( 7 [25\%] vs. $5[12 \%], p=0.16$, respectively). On the other hand, if blanking periods were used (30 days), the recurrence rate was $46.4 \%$ in RFCA vs. $75 \%$ in non-ablated group; $p=0.02$.

Our strong feeling has been based on the assumption that not every early recurrence will lead to late recurrence, and as such, it does not necessarily represent treatment failure. Lack of late recurrences in the ablated group, but their presence in the conservative group, might be responsible for survival benefit (what is also reflected by progressively more divergent with time Kaplan-Meier curves). However, this is only a hypothesis, which has to be confirmed in further studies.

Our concept is supported by an important recent study by Muser et al. [19]. The authors compared the long-term outcomes after VT ablation for ES in patients with nonischemic dilated cardiomyopathy and ischemic cardiomyopathy. Compared to our results, the authors reported a lower incidence of VT recurrence (33\%) in median follow-up 45 months. This difference may be related to the fact that in contrast to our study, they also included patients who underwent endo-epicardial RFCA (22\%). Overall, this outcome may favourably affect long-term outcomes of RFCA. Although VT recurred in 33\% patients after the ablation, a substantial reduction in VT burden was observed in most of these cases. Moreover, the majority of patients $(69 \%)$ with VT recurrence had only isolated ( $\leq 3 \mathrm{VT}$ ) episodes during the 6 months after the RFCA procedure.

The open question remains, what is the rate of clinical to non-clinical arrhythmias among patients with recurrences and what is the impact of non-clinical VT or of early VA recurrence on clinical outcome? 
This issue is even more interesting because, in contrast to our study, Sapp et al. recently reported a significantly lower rate of composite of death as the primary outcome, three or more episodes of ventricular tachycardia within $24 \mathrm{~h}$ (ventricular tachycardia storm) or appropriate ICD shock (59.1\%) in groups of patients with ventricular tachycardia, who were randomly assigned to receive CA with continuation of baseline antiarrhythmic medication vs. $68.5 \%$ of those in the escalated pharmacological therapy group, $p=0.04$ [20]. There was also no significant difference in mortality between the groups [20]. Their results may be explained by several reasons. The authors only conducted their analysis with a follow-up period of $27.9 \pm 17.1$ months in the group of patients with ischaemic cardiomyopathy after myocardial infarction and implanted with ICD, and who had experienced an episode of VT within the 6 months before enrolment. During follow-up, they analysed only the VT episodes and appropriate ICD shock after the 30-day treatment period. There is also lack of data on the percentage of previous RFCA and ES, which may reflect on their outcomes. Notably and surprising is the fact that our study, based on multivariable Cox regression analysis, provides the evidence that the presence of CRT-D is an independent predictor of all-cause mortality in patients with electrical storm. We suppose that this may be attributed to the fact that in contrast to ICD recipients, patients who initially demand the implantation of CRT-D are characterised by increased severity of heart failure, and may be much more exposed to the worsening of heart failure, resulting from multiple high-energy therapies within a short time period.

\subsection{Study limitation}

The first important limitation is the relatively small sample size. However, because of the lack of data surrounding the efficiency of RFCA treatment in ES, our study contributes valuable information. Secondly, although the electrophysiologists who performed RFCA in our study were experienced in the procedure, we cannot rule out that specialised referral centres for endo- and epicardial RFCA could achieve better clinical outcomes. The third limitation is that we also cannot rule out that there might also be some preferences and intrinsic bias among practicing clinicians who perform CA procedures (e.g. preferring patients who are more likely to have a successful outcome for referring to the electrophysiology lab) which may have an influence on differences in the long-term treatment effect of ES in ablated and non-ablative group. The fourth and the most important limitation of our study was the retrospective analysis of short- and long-term outcomes in patients treated because of the occurrence of ES, and its retrospective comparison of RFCA treatment with non-RFCA therapy, not a controlled randomised trial comparing RFCA with other forms of treatment ES. Therefore, our study cannot unambiguously prove that RFCA is more effective than other therapies to enhance survival in patients with ES.

\section{Conclusions}

By comparing long-time effects of ablation to other forms of electrical storm treatment among patients with heart failure and implanted ICD/CRT-D, who had a first episode of electrical storm, a statistically significant reduction in terms of total morality in the group treated with RFCA compared with those of the remaining population was observed.

\section{Compliance with ethical standards}

Conflict of interest Author Morawski Stanislaw has received financial support for attending cardiology congresses from Biotronik.

Author Patrycja Pruszkowska has received consultant fees from Biotronik, Medtronic and St Jude Medical.

Author Sredniawa Beata has received consultant fees from Medtronic Bakken Research Center and has received financial support for attending cardiology congresses from Medtronic, St. Jude Medical, Biotronik and Boston Scientific.

Author Lenarczyk Radoslaw has received consultant fees from Biotronik, Medtronic, St. Jude Medical, Boston Scientific and Boehringer Ingelheim.

Author Kalarus Zbigniew is a member of advisory committee: Boehringer Ingelheim, Amgen and Astra Zeneca. He is a speaker bureau representing the following: Pfizer, Eli Lilly, Boehringer Ingelheim, Abbott and Bayer. He has received a financial support for attending cardiology congresses from Adamed and St. Jude Medical.

Ethical approval All procedures performed in studies involving human participants were in accordance with the ethical standards of the institutional and/or national research committee and with the 1964 Helsinki declaration and its later amendments or comparable ethical standards. For this type of study, formal consent is not required.

Informed consent Informed consent was not required

Open Access This article is distributed under the terms of the Creative Commons Attribution 4.0 International License (http:// creativecommons.org/licenses/by/4.0/), which permits unrestricted use, distribution, and reproduction in any medium, provided you give appropriate credit to the original author(s) and the source, provide a link to the Creative Commons license, and indicate if changes were made.

\section{References}

1. Mosterd A, Hoes AW. Clinical epidemiology of heart failure. Heart. 2007;93:1137-46.

2. Brignole M, Auricchio A, Baron-Esquivias G, et al. 2013 ESC guidelines on cardiac pacing and cardiac resynchronization therapy: the task force on cardiac pacing and resynchronization therapy of the European Society of Cardiology (ESC). Developed in collaboration with the European Heart Rhythm Association (EHRA). Europace. 2013;15:1070-118.

3. McMurray JJ, Adamopoulos S, Anker SD, et al. ESC guidelines for the diagnosis and treatment of acute and chronic heart failure 2012: 
the Task Force for the Diagnosis and Treatment of Acute and Chronic Heart Failure 2012 of the European Society of Cardiology. Developed in collaboration with the Heart Failure Association (HFA) of the ESC. Eur Heart J. 2012;33:1787-847.

4. Braunschweig F, Boriani G, Bauer A, et al. Management of patients receiving implantable cardiac defibrillator shocks: recommendations for acute and long-term patient management. Europace. 2010;12:1673-90.

5. Sesselberg HW, Moss AJ, McNitt S, et al. Ventricular arrhythmia storms in postinfarction patients with implantable defibrillators for primary prevention indications: a MADIT-II substudy. Heart Rhythm. 2007;4:1395-402.

6. Israel CW, Barold SS. Electrical storm in patients with an implanted defibrillator: a matter of definition. Ann Noninvasive Electrocardiol. 2007;12:375-82.

7. Dagres N, Cantù F, Geelen P, Lewalter T, Proclemer A, BlomströmLundqvist C. Current practice of ventricular tachycardia ablation in patients with implantable cardioverter-defibrillators. Europace. 2012;14:135-7.

8. Stevenson WG, Wilber DJ, Natale A, et al. Irrigated radiofrequency catheter ablation guided by electroanatomic mapping for recurrent ventricular tachycardia after myocardial infarction: the multicenter thermocool ventricular tachycardia ablation trial. Circulation. 2008;118:2773-82.

9. Carbucicchio C, Santamaria M, Trevisi N, et al. Catheter ablation for the treatment of electrical storm in patients with implantable cardioverter-defibrillators: short- and long-term outcomes in a prospective single-center study. Circulation. 2008;117:462-9.

10. Guerra F, Shkoza M, Scappini L, Flori M, Capucci A. Role of electrical storm as a mortality and morbidity risk factor and its clinical predictors: a meta-analysis. Europace. 2014;16:347-53.

11. Gatzoulis KA, Andrikopoulos GK, Apostolopoulos T, et al. Electrical storm is an independent predictor of adverse long-term outcome in the era of implantable defibrillator therapy. Europace. 2005; 7:184-92.

12. Exner DV, Pinski SL, Wyse DG, et al. Electrical storm presages nonsudden death: the antiarrhythmics versus implantable defibrillators (AVID) trial. Circulation. 2001;103:2066-71.

13. Nordbeck P, Seidl B, Fey B, Bauer WR, Ritter O. Effect of cardiac resynchronization therapy on the incidence of electrical storm. Int $\mathrm{J}$ Cardiol. 2010;143:330-6.
14. Nayyar S, Ganesan AN, Brooks AG, Sullivan T, Roberts-Thomson $\mathrm{KC}$, Sanders P. Venturing into ventricular arrhythmia storm: a systematic review and meta-analysis. Eur Heart J. 2013;34:560-71.

15. Pedersen CT, Kay GN, Kalman J, et al. EHRA/HRS/APHRS expert consensus on ventricular arrhythmias. Heart Rhythm. 2014;11: e166-96.

16. Aliot EM, Stevenson WG, Almendral-Garrote JM, et al. EHRA/HRS expert consensus on catheter ablation of ventricular arrhythmias: developed in a partnership with the European Heart Rhythm Association (EHRA), a registered branch of the European Society of Cardiology (ESC), and the Heart Rhythm Society (HRS); in collaboration with the American College of Cardiology (ACC) and the American Heart Association (AHA). Heart Rhythm. 2009;6:886-933.

17. Zipes DP, Camm AJ, Borggrefe M, et al. ACC/AHA/ESC 2006 guidelines for management of patients with ventricular arrhythmias and the prevention of sudden cardiac death: a report of the American College of Cardiology/American Heart Association Task Force and the European Society of Cardiology Committee for Practice Guidelines (writing committee to develop guidelines for management of patients with ventricular arrhythmias and the prevention of sudden cardiac death). J Am Coll Cardiol. 2006;48: e247-346.

18. Priori SG, Blomström-Lundqvist C, Mazzanti A, et al. 2015 ESC guidelines for the management of patients with ventricular arrhythmias and the prevention of sudden cardiac death: the task force for the management of patients with ventricular arrhythmias and the prevention of sudden cardiac death of the European Society of Cardiology (ESC). Endorsed by: Association for European Paediatric and Congenital Cardiology (AEPC). Eur Heart J. 2015;36:2793-867.

19. Muser D, Liang JJ, Pathak RR, et al. Long-term outcomes of catheter ablation of electrical storm in nonischemic dilated cardiomyopathy compared with ischemic cardiomyopathy. JACC: Clin Electrophysiol. 2017;371 https://doi.org/10.1016/ j.jacep.2017.01.020.

20. Sapp JL, Wells GA, Parkash R, et al. Ventricular tachycardia ablation versus escalation of antiarrhythmic drugs. $\mathrm{N}$ Engl $\mathrm{J}$ Med. 2016:375:111-21. 